\title{
A replacement for AGOT graphite?
}

\author{
Adam L. Woodcraft ${ }^{\mathrm{a}, 1}$, William D. Duncan ${ }^{\mathrm{b}}$, Peter R. Hastings ${ }^{\mathrm{b}}$ \\ ${ }^{a}$ Department of Physics and Astronomy, University of Wales, Cardiff, P.O. Box 913, Cardiff, CF24 3YB, UK. \\ ${ }^{\mathrm{b}}$ UK Astronomy Technology Centre, Royal Observatory, Edinburgh, Blackford Hill, Edinburgh, EH9 3HJ, UK
}

\begin{abstract}
Pitch bonded graphites are among the best known thermal insulators at sub-Kelvin temperatures. At higher temperatures, however, they are good conductors. Graphite supports can therefore be used to allow a relatively rapid cool-down of structures which must be thermally isolated when cold. The low temperature conductivity of graphites varies by many orders of magnitude depending on the manufacturing process. AGOT graphite is known to be a very good insulator, but is no longer commercially available. We suggest a replacement, and present thermal conductivity measurements demonstrating its suitability.
\end{abstract}

Keywords: graphite; thermal conductivity; support structures

Pitch bonded graphites are among the best known thermal insulators at sub-Kelvin temperatures, but are good conductors at higher temperatures. Graphite supports can therefore be used as a 'passive' heat switch, to allow a relatively rapid cool-down of a structure which must be thermally isolated when cold. AGOT graphite is a pitch bonded graphite which is known to be a very good insulator below $1 \mathrm{~K}$ [1]. However, it is no longer commercially available. Finding a replacement is not straightforward since the conductivity of graphites varies by many orders of magnitude depending on the manufacturing process.

Thermal conductivity measurements [2] above $5 \mathrm{~K}$ on $\mathrm{POCO}^{2}$ AXM-5Q1 graphite show a similar, but slightly lower conductivity than AGOT [3,4], suggesting it as a possible replacement. This material is readily available. We have measured a sample of AXM-5Q

\footnotetext{
1 Corresponding author. E-mail: A.Woodcraft@physics.org 2 POCO graphite, 300 Old Greenwood Rd, Decatur, Texas 76234 USA
}

Physica B, 329-333:1662-1663, 2003 below $1 \mathrm{~K}$. The properties of this material are given in Table 1. AXM-5Q1 is similar, differing only in that it has undergone an extra stage of purification to remove metallic impurities.

Measurements were made using a paramagnetic salt adiabatic demagnetisation refrigerator [5]. A cylindrical sample of AXM-5Q graphite with $25.4 \mathrm{~mm}$ diameter and $6.1 \mathrm{~mm}$ height was glued between copper plates using Stycast 1266 epoxy. The flat ends of the sample were perpendicular to the extrusion axis of the graphite. One of the copper plates was mounted on the demagnetisation stage of the cryostat. The stage

Particle size Pore size Total porosity Apparent density

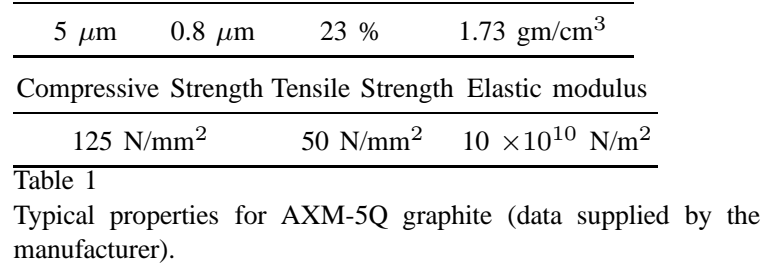

http://www.sciencedirect.com/science/journal/09214526 
contains a calibrated germanium thermometer [5]. A thermometer was mounted on the other plate (the 'hot' plate), while heaters (4.7 M $\Omega$ resistors) were mounted on both plates. The only thermal contact between the plates was via the graphite itself, and through six constantan wires (50 $\mu \mathrm{m}$ diameter), with a negligible calculated heat conduction of $0.1 \mu \mathrm{W}$ at $1 \mathrm{~K}$ [6].

Measurements were made by temperature controlling the stage at various temperatures, and measuring the hot plate temperature as a function of power to the hot plate heater. In each case a linear relationship was seen, providing a good consistency check on the measurements. The gradient of this relationship gives the thermal conductance from the hot plate to the stage. Temperature gradients were kept sufficiently small that the change in conductance along the sample was negligible. The heater on the plate mounted on the stage was used in a similar manner to calculate the conductance from that plate to the stage. The conductance of the graphite sample alone could then be calculated.

The conductance thus measured includes the boundary resistance between the sample and the copper plates. Both the conductance through the thin layer of Stycast [7] and the Kapitza conductance at the boundary [8] should be negligible compared with the graphite conductance. The measured conductance can therefore be taken to be that of the bulk graphite alone.

However, a problem was discovered when, after the measurements, the plates were pulled off the sample. Despite the fact that a large force was required to remove them, it was discovered that on one side the epoxy was absent over approximately $75 \%$ of the area, presumably having been squeezed out during the glueing process. The thermal contact in the 'dry' areas is likely to have been poor. Our measurements thus represent a lower limit on the graphite conductivity, since they include an unknown thermal resistance across the poor contact.

We can obtain an upper limit for conductivity by using the area which was glued well instead of the actual graphite cross-section. This is an upper limit for two reasons. Firstly the actual contact area is probably larger than the $25 \%$ figure used. Secondly, the effective cross section will be larger than the contact area since in this situation the heat flow will not be entirely

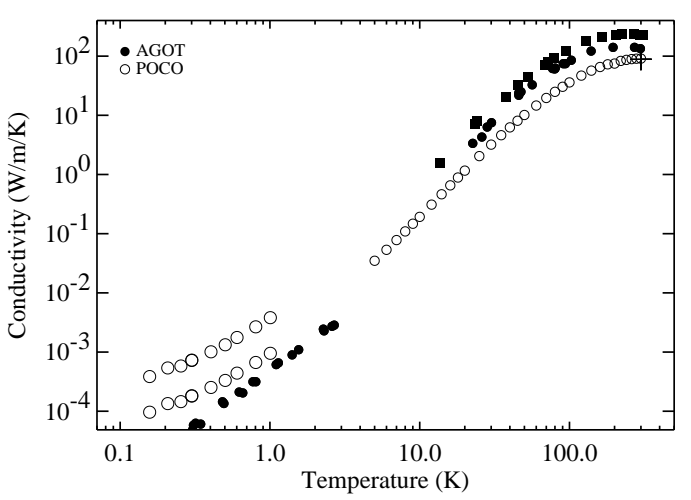

Fig. 1. Measured values for POCO AXM-5Q (open circles, below $1 \mathrm{~K}$ ), showing upper and lower limits as described in the text. The following results from the literature are also plotted for comparison: POCO AXM-5Q1 [2] (open circles, above $1 \mathrm{~K}$ ), POCO AXM-5Q [estimated value supplied by manufacturer] (cross), AGOT [1] (closed circles, below $10 \mathrm{~K}$ ), AGOT [3] (closed circles, above $10 \mathrm{~K}$ ), AGOT [4] (closed boxes).

perpendicular to the sample faces.

The results are shown in Fig. 1. Our measurements appear to be consistent with those in the literature for higher temperatures. While not as good as AGOT graphite, the POCO graphite is still a very good insulator below $1 \mathrm{~K}$, and has a conductivity which reduces by approximately five orders of magnitude between $300 \mathrm{~K}$ and $1 \mathrm{~K}$. It therefore makes a suitable, and easily available, material for a passive heat switch.

\section{Acknowledgement}

We thank POCO for the donation of the graphite sample, and M. J. Griffin for useful comments.

\section{References}

[1] D. O. Edwards, R. E. Sarwinski, P. Seligmann, J. T. Tough, Cryogenics 8 (1968) 392-393.

[2] J. G. Hust, Fine-grained, isotropic graphite for use as NBS (National Bureau of Standards) thermophysical property RM's from 5 to $2500 \mathrm{~K}$ (Final report), National Bureau of Standards, Boulder, Colorado, 1984, NBS/SP-260/89.

[3] W. W. Tyler, J. A. C. Wilson, Physical Review 89 (1953) 870-875. 
[4] A. W. Smith, N. S. Rasor, Physical Review 104 (1956) 885891.

[5] A. L. Woodcraft, R. V. Sudiwala, M. J. Griffin, E. Wakui, B. Maffei, C. E. Tucker, C. V. Haynes, F. Gannaway, P. A. R. Ade, J. J. Bock, A. D. Turner, S. Sethuraman, J. W. Beeman, International Journal of Infrared and Millimeter Waves 23 (2002) 575-595.

[6] G. K. White, Experimental techniques in low-temperature physics, Oxford: Clarendon Press, 1979.

[7] F. Pobell, Matter and Methods at Low Temperatures, Springer, 1992.

[8] A. C. Anderson, R. E. Peterson, Cryogenics 10 (1970) 430-3. 